\title{
Molecular Docking Studies Involving the Inhibitory Effect of Gymnemic Acid, Trigonelline and Ferulic Acid, the Phytochemicals with Antidiabetic Properties, on Glycogen Synthase Kinase 3 ( $\alpha$ and $\beta$ )
}

\author{
V. Roshana Devi, C. Sharmila, S. Subramanian* \\ Department of Biochemistry, University of Madras, Guindy Campus, Chennai - 600 025, Tamilnadu, India.
}

\begin{tabular}{l}
\hline ARTICLE INFO \\
\hline Article history: \\
Received on: 09/02/2018 \\
Accepted on: $18 / 03 / 2018$ \\
Available online: $29 / 04 / 2018$
\end{tabular}

\section{Key words:}

Diabetes mellitus, phytochemicals, glycogen synthase kinase 3 , glycogen metabolism, in silico studies.

\begin{abstract}
Type 2 diabetes mellitus (T2DM) accounts for more than $90 \%$ of the diabetic population and its prevalence is linked to obesity-induced insulin resistance followed by a relative rate of insulin secretion from the $\beta$-cells of the pancreas. Several drugs are available to maintain normoglycemia in diabetic individuals through the regulation of biological functions such as controlling the absorption of glucose in the intestine, increasing the insulin sensitivity, improvement in the secretion of insulin, prevention of excessive breakdown of glucose as well as gluconeogenesis and enhancement of glycogen synthesis. Since, most of the currently available drugs elicit undesirable side effects in addition to the development of resistance after prolonged use, the search for lead molecules preferably from plant origin still continues. Recently, we have formulated a mixture containing three phytochemicals from medicinal plants namely Gymnemic acid, Trigonelline and Ferulic acid in the ratio of 2:3:1 and systematically evaluated its antidiabetic efficacy in type 2 diabetes in rats. Glycogen metabolism plays an important role in the regulation of glucose homeostasis and it is controlled by the two rate-limiting enzymes namely glycogen synthase and glycogen phosphorylase. The activity of glycogen synthase is regulated by glycogen synthase kinase, a negative regulator of insulin signaling pathway. GSK-3 subsists in two similarly associated isoforms namely GSK-3 $\alpha$ and GSK-3 $\beta$ with the molecular weight of 51 $\mathrm{kDa}$ and $47 \mathrm{kDa}$, respectively. They possess almost indistinguishable affinities for their substrates and their biological role. The present study is aimed to perform molecular docking studies involving the inhibitory effect of individual phytochemicals with antidiabetic properties on Glycogen synthase kinase-3 $(\alpha \& \beta)$. The binding energy obtained for GA, TG and FA for GSK-3 $\alpha$ is found to be $-5.33,-6.85$, and $-7.0 \mathrm{Kcal} / \mathrm{Mol}$, respectively. Likewise, the binding energy calculated for GA, TG, and FA for GSK-3 $\beta$ is found to be $-4.69,-5.51$, and $-5.05 \mathrm{Kcal} / \mathrm{Mol}$, respectively. The data obtained evidenced the inhibitory role of GTF on the activity of GSK isoforms in the regulation of glycogen metabolism.
\end{abstract}

\section{INTRODUCTION}

Glycogen synthase kinase-3 is a multi-tasking cytosolic serine/threonine protein kinase, originally identified by Embi et al. (1980) from the rabbit skeletal muscle as a regulator of glycogen synthase (GS). In contrast to other kinases, the action of GSK-3 inhibits glycogen synthase activity. GSK-3 subsists in two similarly associated isoforms namely GSK-3 $\alpha$ and GSK-3 $\beta$ with

\section{${ }^{*}$ Corresponding Author}

Dr. S. Subramanian, Professor, Department of Biochemistry, University of Madras, Guindy Campus, Chennai - 600 025, Tamilnadu, India.

E-mail:subbus2020@yahoo.co.in the molecular weight of $51 \mathrm{kDa}$ and $47 \mathrm{kDa}$, respectively. They possess almost indistinguishable affinities for their substrates and their biological role (Stambolic and Woodgett, 1994).

Type 2 diabetes mellitus (T2DM) accounts for more than $90 \%$ of the diabetic population and its prevalence is linked to obesity-induced insulin resistance followed by a relative rate of insulin secretion from the $\beta$-cells of the pancreas. Interestingly, the elevated activities of GSK-3 have been reported in diabetic and obese strains of rats/mice (Eldar-Finkelman et al., 1999). The activity of glycogen synthase is regulated by glycogen synthase kinase, a negative regulator of insulin signaling pathway and its activity is implicated in various pathological conditions including 
type 2 diabetes, malignant cells, and chronic inflammatory disorders. It phosphorylates and inhibits the functioning of two key targets of the insulin pathway namely IRS-1 and glycogen synthase. Insulin receptors in the peripheral tissues provoke a cascade of signaling pathways that result in the activation of $\mathrm{Akt}$ which subsequently gets phosphorylates to inhibit the activity of GSK3 (Cross et al., 1995). Thus, insulin decreases the activity of GSK-3 (Lawlor and Alessi, 2001). GSK is also been implicated in ER stress in the $\beta$ cells and decrease the activity of GSK thereby protect the cells from death (Srinivasan et al., 2005).

The N-terminal phosphorylation of GSK-3 $\alpha$ at Serine 21 and GSK-3 $\beta$ at Serine 9 elicits an inhibitory effect in the regulation of GSK function. Increased expression of GSK 3 was reported in the skeletal muscle of T2DM patients evidencing its role in insulin signaling (Nikoulina et al., 2000).

Several synthetic GSK-3 inhibitors have been established to date. Very few studies have been conducted on plant-derived compounds with pharmacoinformatics elucidation for the identification of novel therapeutics for diabetes (Ahmed et al., 2014). However, the clinical trials are limited due to their cytotoxicity, low pharmacokinetic and pharmacodynamic characteristics. Hence, the development of effective GSK-3 inhibitors preferably from plant sources having a discrete mechanism of action on its substrates and the structure-based studies have provided the vital clues on the interactions shown by selective and non-selective ATP-competitive GSK-3 inhibitors (Wauwe et al., 2003). Recently, we have reported the computeraided approach on aldose reductase inhibitory potential of gymnemic acid, trigonelline and ferulic acid (Roshana Devi and Subramanian, 2017).

Plant-derived secondary metabolites have a wide array of therapeutic efficacy (Atanasov et al., 2015; Ota and Ulrih, 2017; Liu et al., 2017). Gymnemic acid, a sweet taste suppressing glycoside, is the major phytoingredient isolated from the leaves of Gymnema Sylvestre. Trigonelline, a pyridine alkaloid, isolated from the seeds of fenugreek (Trigonella foenum-graecum) and known for its hypoglycemic and hypolipidemic properties. Ferulic acid, isolated from the oleo gum resin of Ferula asafetida has received great attention in oriental research due to its significant antioxidant properties.

The present study involves the computer-aided molecular docking approach on the inhibition of GSK-3 $\alpha$ and GSK-3 $\beta$ activity by the above phytoingredients.

\section{MATERIALS AND METHODS}

\section{Ligand preparation}

The phytochemicals such as Gymnemic acid, Trigonelline, and Ferulic acid were considered as ligand molecules. The phytochemicals were constructed using Chemsketch and then converted into PDB file format by adding the hydrogen bonds.

\section{Preparation of receptor protein}

Crystal structure of glycogen synthase kinase-3 was retrieved from RCSB PDB. Preparation of glycogen synthase kinase-3 with the Auto Dock Tools involves the addition of hydrogen atoms to the target enzyme for the preparation of protein docking simulation.

\section{Molecular docking using Auto Dock}

Auto Dock Tools were used to study the docking simulations (Trott and Olson, 2010). Auto Dock 4.2 is used to study the molecular interactions between the phytochemical ligands such as Gymnemic acid, Trigonelline and Ferulic acid and the enzyme receptor, glycogen synthase kinase-3.

Auto Dock requires pre-calculated grid maps, one for each type of atom present in the flexible molecules being docked and its stores the potential energy arising from the interaction with rigid macromolecules. This grid must surround the region of interest in the rigid macromolecule. The grid box size was set at 126,126 , and $126 \AA$ (x,y, and $\mathrm{z}$ ) to include all the amino acid residues which are present in rigid macromolecules. Auto Grid 4.2 Program, supplied with Auto Dock 4.2 was used to produce grid maps. The spacing between grid points was 0.375 angstroms.

Auto Dock offers a variety of search algorithms to explore a given docking problem. In the present study, Lamarckian Genetic Algorithm (LGA) was chosen search for the best conformers. During the docking process, a maximum of 10 conformers was considered. The population size was set to 150 and the individuals were initialized randomly. The maximum number of energy evaluation was set to 500000, the maximum number of generations 1000 , the maximum number of a top individual that automatically survived set to 1 , the mutation rate of 0.02 , the crossover rate of 0.8 , Step sizes were $0.2 \AA$ for translations, $5.0^{\circ}$ for quaternions and $5.0^{\circ}$ for torsions. Cluster tolerance $0.5 \AA$, external grid energy 1000.0, max initial energy 0.0, max number of retries 10000 and 10 LGA runs were performed.

Auto Dock results were analyzed to study the interactions and the binding energy of the docked structure. It was run several times to get various docked conformations and to analyze predicted docking energy. The best ligand-receptor structure from the docked structures was chosen based on the lowest energy and minimal solvent accessibility of the ligand. The docking results were visualized using the Accelrys Visualizer discovery studio tool.

\section{RESULTS AND DISCUSSION}

In silico molecular docking is a method with predicts the preferred orientation of a molecule to a second when they allowed for binding to each other to form a stable compound (Lengauer and Rarey, 1996). However, the knowledge of the preferential orientation is essential to predict the strength of association or the affinity binding between the two molecules based on their scoring function.

Essentially, the molecular docking is a tricky process comprising of two parts namely the prediction and orientation of the ligand and the affinity towards its target (Bissantz et al., 2000). The relative orientation of the two interacting molecules such as proteins, nucleic acids, carbohydrates, and lipids play a major role in signal transduction which in turn found useful for predicting the strength and the type of signal generated. Thus, the lowest binding energy observed during the molecular docking plays an important role in rational drug designing (Kitchen et al., 2004).

The identification and elucidation of GSK-3 in the regulation of various metabolic pathways result in the search for suitable GSK inhibitors as candidates for the prevention and 
treatment of several chronic diseases (Mohammad et al., 2008). So far, a variety of molecules have been synthesized to inhibit the glycogen synthase kinase activity. However, none is found to be ideal due to several drawbacks. Hence, the search for novel GSK inhibitors preferably from plant origin with improved efficacy and safety continues for the development of antidiabetic drugs. The molecular structures of the phytoligands used in the present study were presented in Figure 1.

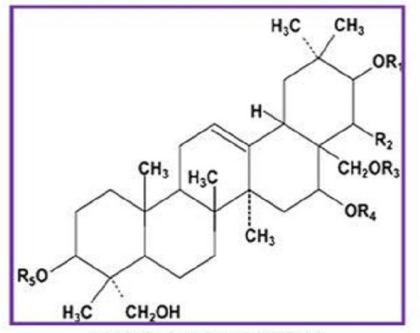

GYMNEMIC ACID

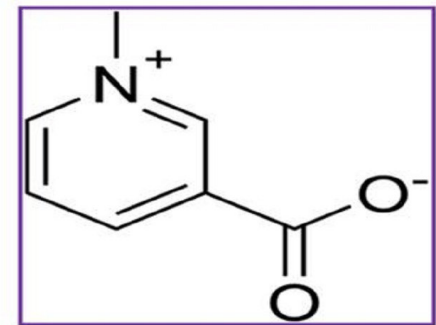

TRIGONEUNE

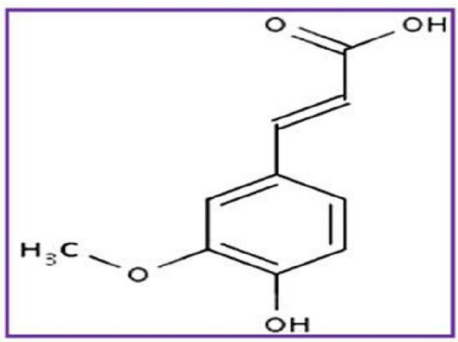

FERULCACID

Fig. 1: Molecular structures of phytoligands.
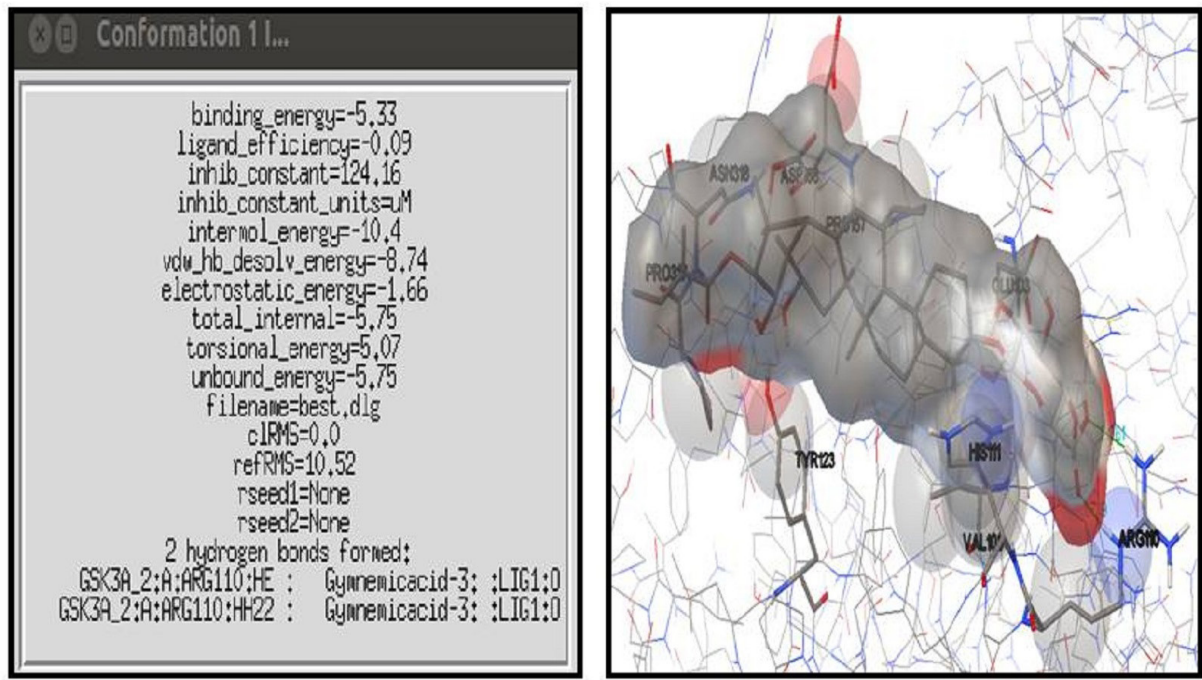

Fig. 2: Docking conformation of Gymnemic acid with Glycogen synthase kinase $3 \alpha$ using Auto Dock.

\section{MOLECULAR DOCKING STUDIES OF GYMNEMIC ACID, TRIGONELLINE AND FERULIC ACID ON GLYCOGEN SYNTHASE KINASE 3 (A \& B)}

\section{Inhibition of Glycogen synthase kinase 3 activity by} Gymnemic acid

Figure 2 exemplifies the docking conformation of gymnemic acid with glycogen synthase kinase $3 \alpha$. The interactions of hydrogen bonds between glycogen synthase kinase- $3 \alpha$ and gymnemic acid was visualized as Figure 3. Table 1 represents the docking energy and the bonding distance between gymnemic acid and glycogen synthase kinase $3 \alpha$. This study shows that the gymnemic acid with glycogen synthase kinase $3 \alpha$ complex is stabilized by the hydrogen bonds of bond length $2.43,2.98,1.87,3.14,2.61$, and $3.05 \AA$ with the residues TYR123, TYR123, TYR123, VAL108 ARG110, and ARG110 of GSK $3 \alpha$, respectively. The interactions that play a significant role in the determination of binding energy and stability of these receptor-ligand complexes were recognized as hydrogen bonds. 
The binding energy of the lowest energy conformer of gymnemic acid with glycogen synthase kinase $3 \alpha$ complex was calculated computationally and found to be $-5.33 \mathrm{Kcal} / \mathrm{mol}$.

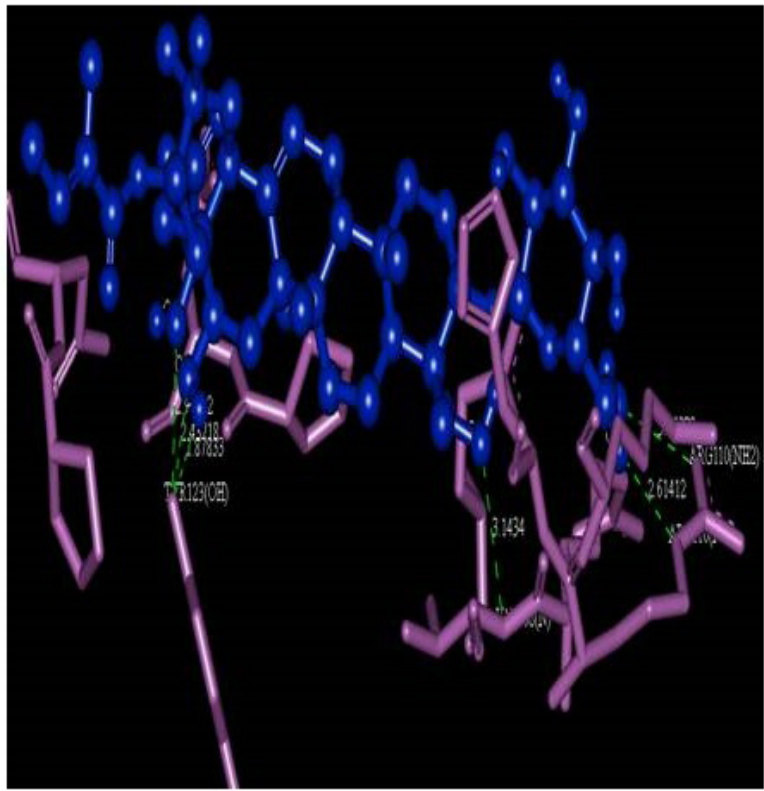

Fig. 3: Hydrogen bonds interaction between GSK3 $\alpha$ and gymnemic acid using Accelrys Discovery Studio Visualizer.
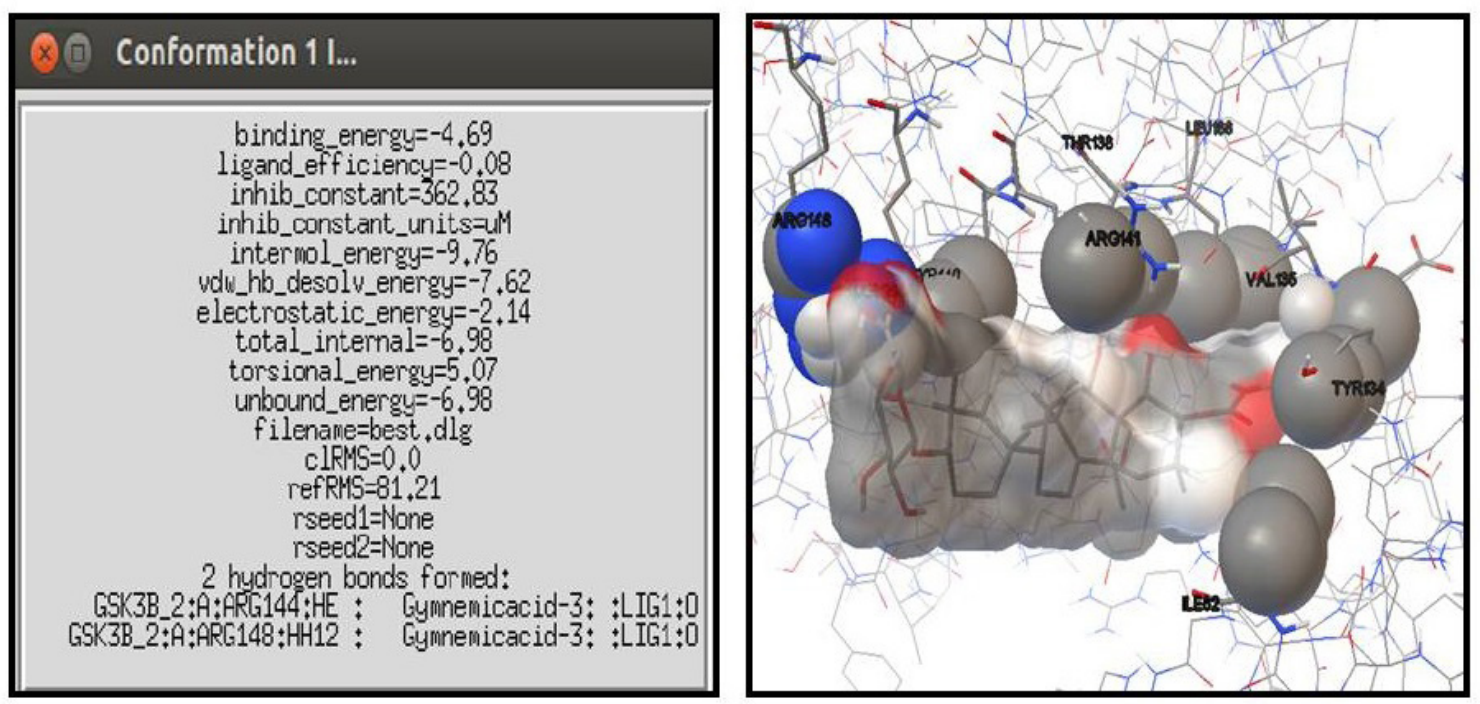

Fig. 4: Docking conformation of Gymnemic acid with Glycogen synthase kinase $3 \beta$ using Auto Dock.

Figure 4 illustrates the docking conformation of gymnemic acid with glycogen synthase kinase $3 \beta$. The interactions of hydrogen bonds between glycogen synthase kinase and gymnemic acid was visualized as Figure 5. Table 2 shows the docking energy and the bonding distance between gymnemic acid and glycogen synthase kinase $3 \beta$. The amino acids present in the active site of glycogen synthase kinase $3 \beta$ interacts with gymnemic acid are found to be ARG148, ARG148 ARG148, ARG144, and ARG144. The docking complex is stabilized by the hydrogen bonds of distance was 2.68, 3.16, 2.85, 2.80, 2.76, and $3.05 \AA$. The docking energy for gymnemic acid with Glycogen synthase kinase $3 \beta$ was found to be $-4.69 \mathrm{Kcal} / \mathrm{Mol}$.

\section{Inhibition of Glycogen synthase kinase 3 activity by Trigonelline}

Docking conformation of Trigonelline with glycogen synthase kinase $3 \alpha$ was depicted as Figure 6 . The interactions of hydrogen bonds between glycogen synthase kinase $3 \alpha$ and 
Trigonelline were visualized in Figure 7. Table 3 represents the docking energy and the bonding distance for Trigonelline with glycogen synthase kinase $3 \alpha$. The amino acids present in the active site of glycogen synthase kinase $3 \alpha$ interacts with Trigonelline are found to be ARG62, ARG62, LYS147, VAL180, ARG146, ARG146, and ARG146 and the hydrogen bonding distance between trigonelline and GSK $3 \alpha$ were found to be 2.87 , $2.95,2.83,3.05,2.92,3.18$, and $2.84 \AA$. The docking energy for Trigonelline with Glycogen synthase kinase $3 \alpha$ was calculated computationally and found to be $-6.85 \mathrm{Kcal} / \mathrm{Mol}$.

Table 1: Docking energy for Gymnemic acid with Glycogen synthase kinase $3 \alpha$.

\begin{tabular}{|c|c|c|c|c|}
\hline \multicolumn{2}{|c|}{ GSK3a } & \multirow{2}{*}{ Gymnemic Acid } & \multirow{2}{*}{ Distance (Á) } & \multirow{2}{*}{$\begin{array}{l}\text { Docking Energy } \\
\text { (Kcal/Mol) }\end{array}$} \\
\hline Residue & Atom & & & \\
\hline TYR123 & $\mathrm{OH}$ & $\mathrm{O}$ & 2.43 & \multirow{6}{*}{-5.33} \\
\hline TYR123 & $\mathrm{OH}$ & $\mathrm{O}$ & 2.98 & \\
\hline TYR123 & $\mathrm{OH}$ & $\mathrm{H}$ & 1.87 & \\
\hline VAL108 & $\mathrm{N}$ & $\mathrm{O}$ & 3.14 & \\
\hline ARG110 & NE & $\mathrm{O}$ & 2.61 & \\
\hline ARG110 & $\mathrm{NH} 2$ & $\mathrm{O}$ & 3.05 & \\
\hline
\end{tabular}

Table 2: Docking energy for Gymnemic acid with Glycogen synthase kinase $3 \beta$.

\begin{tabular}{|c|c|c|c|c|}
\hline \multicolumn{2}{|c|}{ GSK3及 } & \multirow{2}{*}{ Gymnemic Acid } & \multirow{2}{*}{ Distance (Á) } & \multirow{2}{*}{$\begin{array}{l}\text { Docking Energy } \\
\text { (Kcal/Mol) }\end{array}$} \\
\hline Residue & Atom & & & \\
\hline ARG148 & NH1 & $\mathrm{O}$ & 2.68 & \\
\hline ARG148 & $\mathrm{NH} 2$ & $\mathrm{O}$ & 3.16 & \\
\hline ARG148 & $\mathrm{NH} 2$ & $\mathrm{O}$ & 2.85 & -4.69 \\
\hline ARG144 & $\mathrm{NH} 2$ & $\mathrm{O}$ & 2.80 & \\
\hline ARG144 & $\mathrm{NE}$ & $\mathrm{O}$ & 2.76 & \\
\hline
\end{tabular}

Table 3: Docking energy for trigonelline with Glycogen synthase kinase $3 \alpha$.

\begin{tabular}{|c|c|c|c|c|}
\hline \multicolumn{2}{|c|}{ GSK3a } & \multirow{2}{*}{ Trigonelline } & \multirow{2}{*}{ 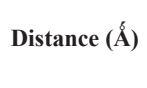 } & \multirow{2}{*}{$\begin{array}{c}\text { Docking Energy } \\
\text { (Kcal/Mol) }\end{array}$} \\
\hline Residue & Atom & & & \\
\hline ARG62 & NH1 & $\mathrm{O}$ & 2.87 & \\
\hline ARG62 & $\mathrm{NH} 2$ & $\mathrm{O}$ & 2.95 & \\
\hline LYS147 & $\mathrm{NZ}$ & $\mathrm{O}$ & 2.83 & \\
\hline VAL180 & $\mathrm{N}$ & $\mathrm{O}$ & 3.05 & -6.85 \\
\hline ARG146 & $\mathrm{NH} 2$ & $\mathrm{O}$ & 2.92 & \\
\hline ARG146 & $\mathrm{NH} 2$ & $\mathrm{O}$ & 3.18 & \\
\hline ARG146 & NH1 & $\mathrm{O}$ & 2.84 & \\
\hline
\end{tabular}

The docking conformation of Trigonelline with glycogen synthase kinase $3 \beta$ is illustrated in Figure 8 . The interactions of hydrogen bonds between glycogen synthase kinase and Trigonelline were visualized as Figure 9. Table 4 represents the docking energy and the bonding distance for Trigonelline with glycogen synthase kinase $3 \beta$. The amino acids present in the active site of glycogen synthase kinase $3 \beta$ interacts with Trigonelline are found to be ARG180, ARG180, ARG180, LYS205, and ARG96. The docking Trigonelline with glycogen synthase kinase $3 \beta$ complex was stabilized by hydrogen bonds interaction and the bonding distance was found to be 3.19, 3.16, 2.82, 2.80, and 2.99 $\AA$. The binding energy for Trigonelline with Glycogen synthase kinase $3 \beta$ was found to be $-5.51 \mathrm{Kcal} / \mathrm{Mol}$.

Table 4: Docking energy for trigonelline with Glycogen synthase kinase $3 \beta$.

\begin{tabular}{|c|c|c|c|c|}
\hline \multicolumn{2}{|c|}{ GSK3 $\beta$} & \multirow{2}{*}{ Trigonelline } & \multirow{2}{*}{ Distance (Á) } & \multirow{2}{*}{$\begin{array}{l}\text { Docking Energy } \\
\text { (Kcal/Mol) }\end{array}$} \\
\hline Residue & Atom & & & \\
\hline ARG180 & NH1 & $\mathrm{O}$ & 3.19 & \\
\hline ARG180 & NH2 & $\mathrm{O}$ & 3.16 & \\
\hline ARG180 & $\mathrm{NH} 2$ & $\mathrm{O}$ & 2.82 & -5.51 \\
\hline LYS205 & NZ & $\mathrm{O}$ & 2.80 & \\
\hline ARG96 & NH1 & $\mathrm{O}$ & 2.99 & \\
\hline
\end{tabular}

Table 5: Docking energy for ferulic acid with Glycogen synthase kinase $3 \alpha$.

\begin{tabular}{|c|c|c|c|c|}
\hline \multicolumn{2}{|c|}{ GSK3 $\alpha$} & \multirow{2}{*}{ Ferulic Acid } & \multirow{2}{*}{ Distance (Á) } & \multirow{2}{*}{$\begin{array}{l}\text { Docking Energy } \\
\text { (Kcal/Mol) }\end{array}$} \\
\hline Residue & Atom & & & \\
\hline SER169 & $\mathrm{OH}$ & $\mathrm{H}$ & 2.07 & \\
\hline SER169 & $\mathrm{OH}$ & $\mathrm{H}$ & 3.02 & \\
\hline ARG146 & NH2 & $\mathrm{O}$ & 3.04 & \\
\hline VAL180 & $\mathrm{N}$ & $\mathrm{O}$ & 2.95 & -7.0 \\
\hline LYS171 & $\mathrm{NZ}$ & $\mathrm{O}$ & 3.03 & \\
\hline LYS171 & $\mathrm{NZ}$ & $\mathrm{O}$ & 3.00 & \\
\hline ARG62 & NH2 & $\mathrm{O}$ & 2.98 & \\
\hline
\end{tabular}

\section{Inhibition of Glycogen synthase kinase 3 activity by Ferulic} acid

Figure 10 exemplifies the docking conformation of ferulic acid with glycogen synthase kinase $3 \alpha$. The interactions of hydrogen bonds between glycogen synthase kinase $3 \alpha$ and ferulic acid were visualized using Accelrys discovery studio visualizer (Figure 11). Table 5 represents the docking energy and the bonding distance for ferulic acid with glycogen synthase kinase $3 \alpha$. The amino acids present in the active site of glycogen synthase kinase $3 \alpha$ interacts with ferulic acid are found to be SER169, SER169, ARG146, VAL180, LYS171, LYS171, and ARG62. GSK $3 \alpha$ and ferulic acid were stabilized by the hydrogen bonds. The bonding distance between ferulic acid with glycogen synthase kinase $3 \alpha$ was found to be $2.07,3.02,3.04,2.95,3.03,3.00$, and $2.98 \AA$. The docking energy for ferulic acid with Glycogen synthase kinase $3 \alpha$ was found to be $-7.0 \mathrm{Kcal} / \mathrm{Mol}$.

Figure 12 illustrates the docking conformation of ferulic acid with glycogen synthase kinase $3 \beta$. The interactions of hydrogen bonds between glycogen synthase kinase and ferulic acid were visualized as Figure 13. Table 6 represents the docking energy and the bonding distance for ferulic acid with glycogen synthase kinase $3 \beta$. Trigonelline with glycogen synthase kinase $3 \beta$ 
complex is stabilized by the hydrogen bonds of bond length 2.56 , 2.78 and $1.84 \AA$ with the residues TYR127, TYR127, and SER66 of GSK $3 \beta$, respectively. The binding energy for ferulic acid with Glycogen synthase kinase $3 \beta$ was found to be $-5.05 \mathrm{Kcal} / \mathrm{Mol}$.
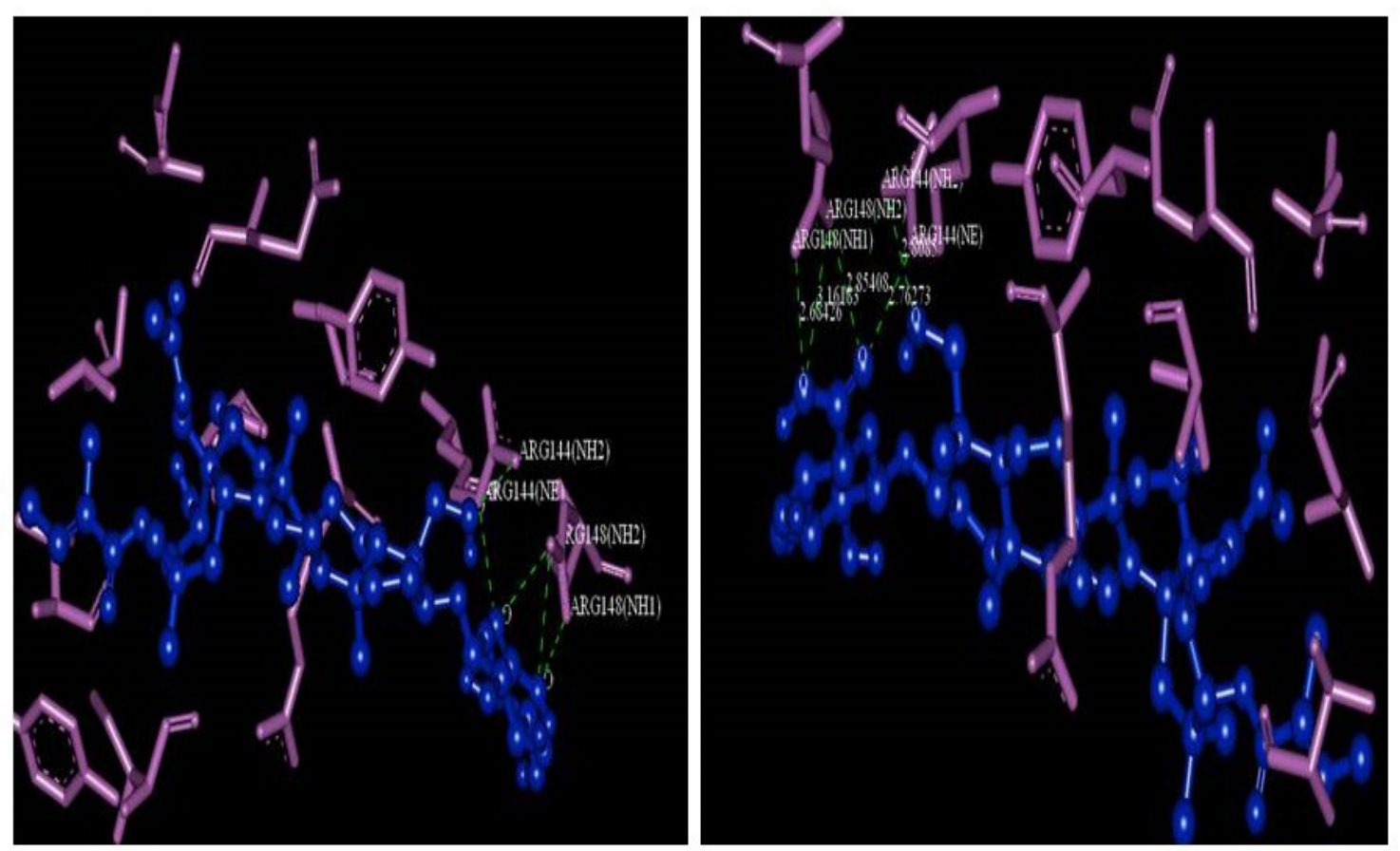

Fig. 5: Hydrogen bonds interaction between GSK3 $\beta$ and Gymnemic acid using Accelrys Discovery Studio Visualizer.
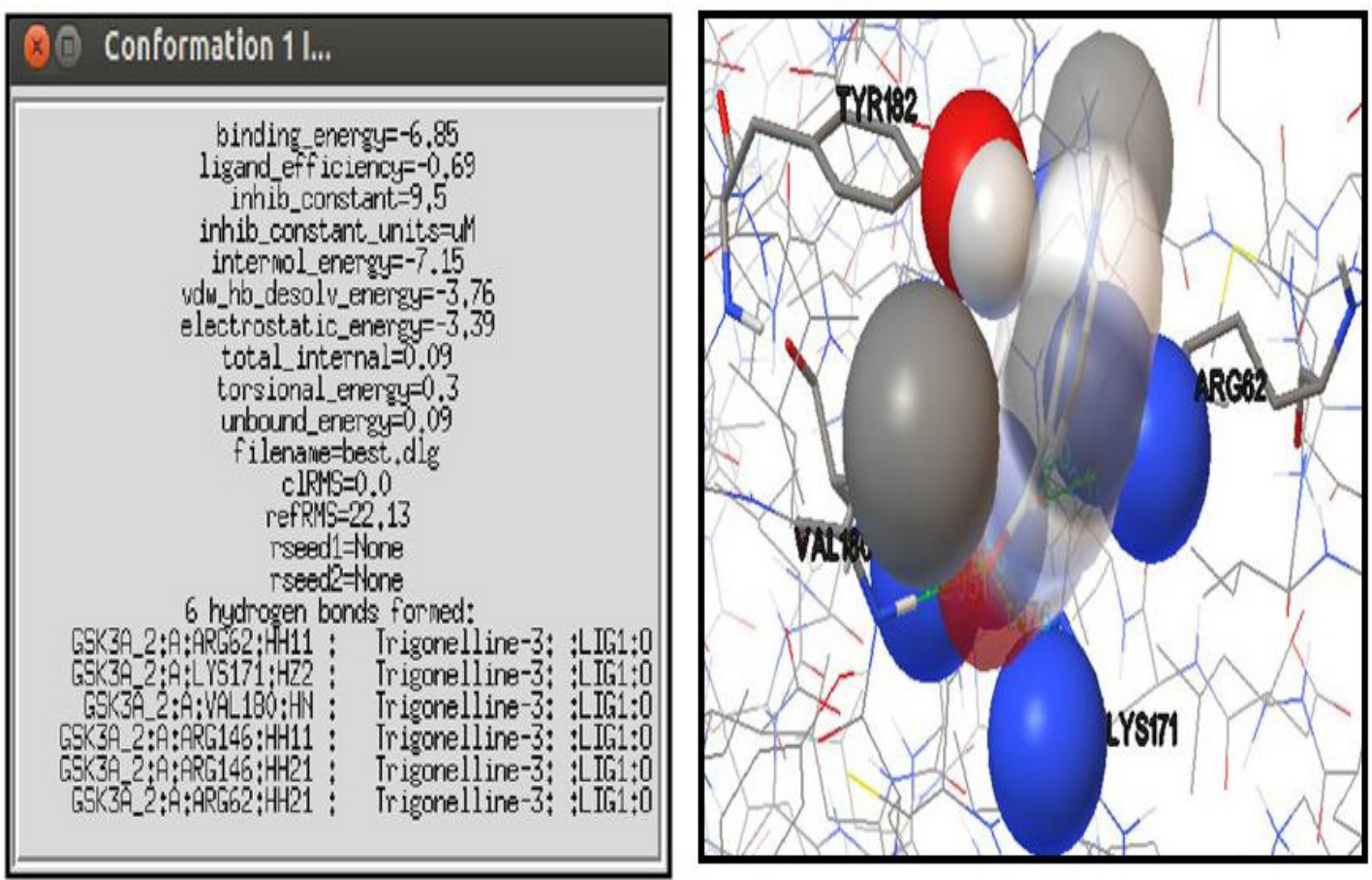

Fig. 6: Docking conformation of trigonelline with Glycogen synthase kinase $3 \alpha$ using Auto Dock. 

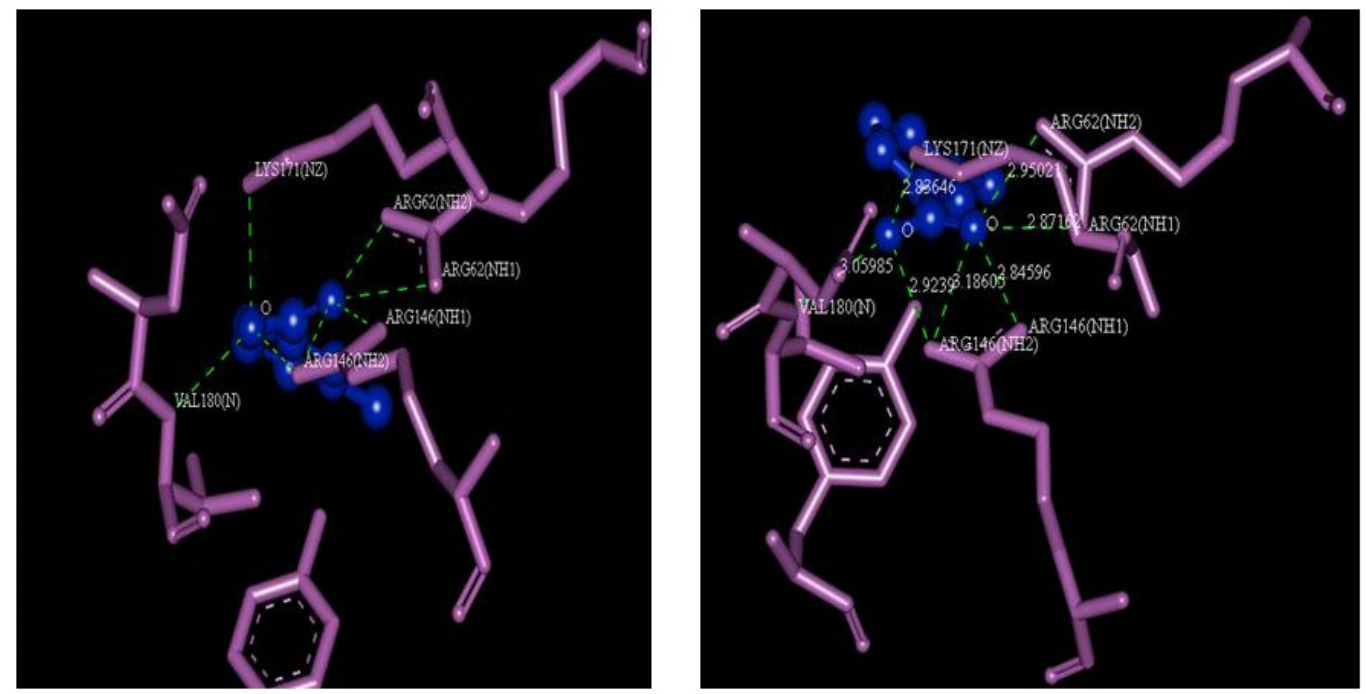

Fig. 7: Hydrogen bonds interaction between GSK3 $\alpha$ and trigonelline using Accelrys Discovery Studio Visualizer.
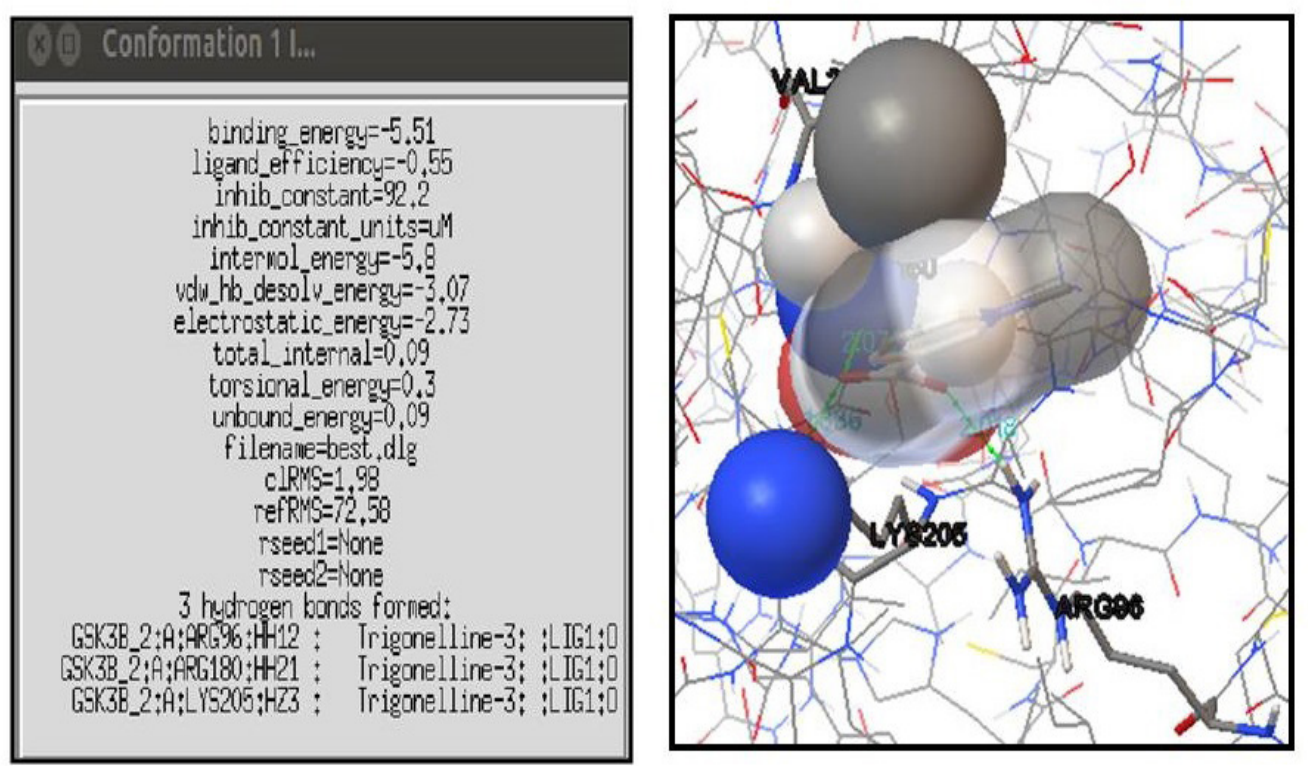

Fig. 8: Docking conformation of trigonelline with Glycogen synthase kinase $3 \beta$ using Auto Dock.

Table 6: Docking energy for ferulic acid with Glycogen synthase kinase $3 \beta$.

\begin{tabular}{ccccc}
\hline \multicolumn{2}{c}{ GSK3 } & Ferulic Acid & Distance (Á) & $\begin{array}{c}\text { Docking } \\
\text { Energy (Kcal/Mol) }\end{array}$ \\
\cline { 1 - 2 } Residue & Atom & & & \\
\hline TYR127 & $\mathrm{N}$ & $\mathrm{O}$ & 2.56 & -5.05 \\
TYR127 & $\mathrm{N}$ & $\mathrm{O}$ & 2.78 & \\
SER66 & $\mathrm{O}$ & $\mathrm{H}$ & 1.84 & \\
\hline
\end{tabular}

The overall binding energies between phytoligands and GSK- $3 \alpha$ and $3 \beta$ were depicted in Table 7 . Table 8 depicts the inhibition constant of Gymnemic acid, Trigonelline and Ferulic acid with Glycogen synthase kinase $3 \alpha$ and $3 \beta$. Intermolecular energies of Gymnemic acid, Trigonelline and Ferulic acid with Glycogen synthase kinase $3 \alpha$ and $3 \beta$ were shown in Table 9.

The parameters analyzed in the present study include hydrogen bond interactions, $\Pi-\Pi$ interactions, binding energy, active site residues and orientation of the phytochemicals with the receptors. These are considered as important indices of molecular docking (Madeswaran et al., 2011). If a compound shows lesser binding energy, it implies that the compounds have higher activity. The extent of forces of attraction or repulsion between the ligand and the receptor depends on the binding or intermolecular energy (Madeswaran et al., 2012). Accordingly, the results of the present study confirmed that the decrease in the binding energy, as well as 
inhibition constant for the three bioactive compounds (ligands), is responsible for their GSK inhibitory activity. In conclusion, the inhibitory role of the gymnemic acid, trigonelline, and ferulic acid on the activity of GSK was proved using molecular docking simulation.
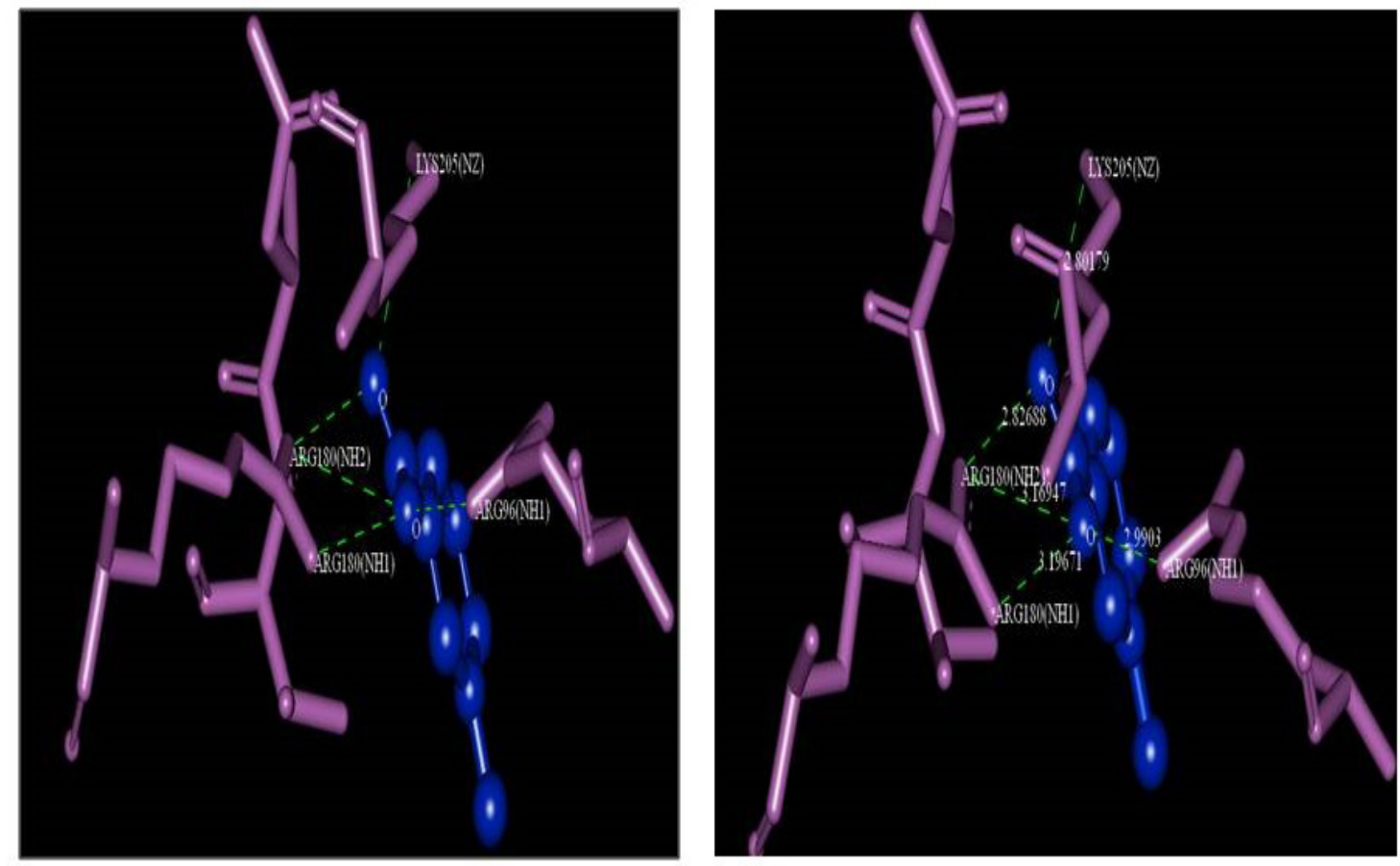

Fig. 9: Hydrogen bonds interaction between GSK3 $\beta$ and trigonelline using Accelrys Discovery Studio Visualizer.
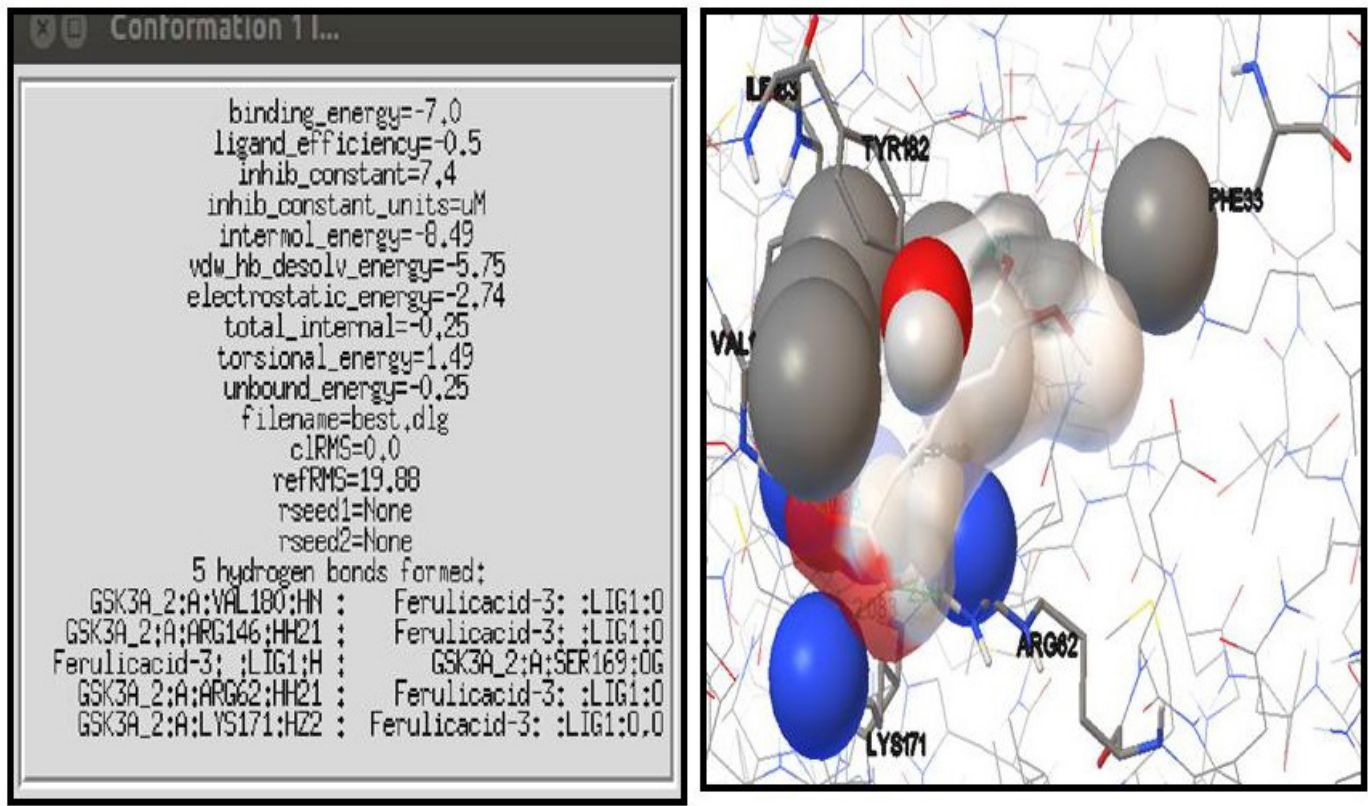

Fig. 10: Docking conformation of ferulic acid with Glycogen synthase kinase $3 \alpha$ using Auto Dock.

Middha et al. (2013) reported that the binding interactions exhibited by the compounds of Hippophae inhibit the activity of GSK-3 $\beta$. In 2013, Shen and Lu reported that the citrus flavonoids inhibit the GSK-3 $\beta$ activity. Quinazoline derivatives 
inhibit the activity of GSK-3 and can be utilized for designing of novel GSK-3 $\beta$ inhibitors (Sangu et al., 2014). Our findings were in accordance with the reported results. Gymnemic acid, Trigonelline, and Ferulic acid possess potential GSK inhibitory binding pockets and it may be due to the presence of chemical structure and $-\mathrm{OH}$ functional groups present in the bioactive compounds, which might be responsible for the inhibitory activity of GSK-3.
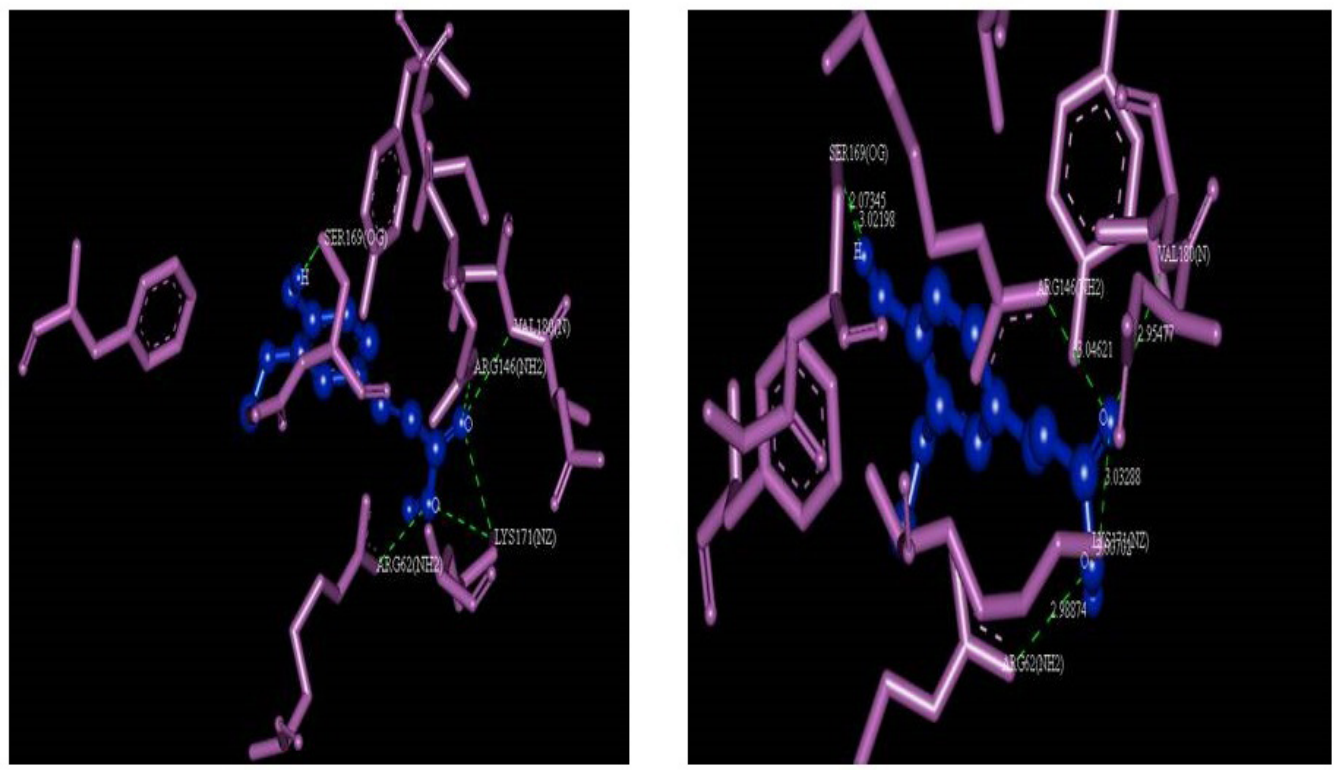

Fig. 11: Hydrogen bonds interactions between GSK3 $\alpha$ and ferulic acid using Accelrys Discovery Studio Visualizer.
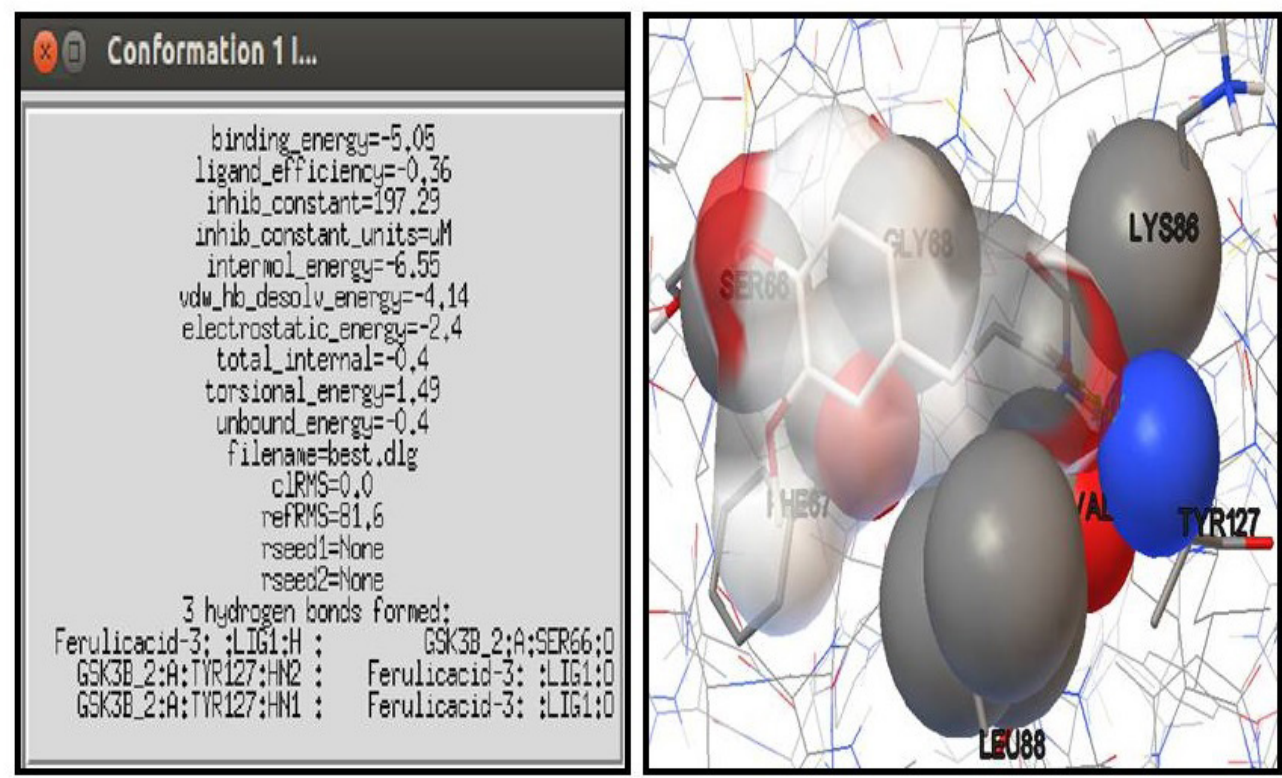

Fig. 12: Docking conformation of ferulic acid with Glycogen synthase kinase $3 \beta$ using Auto Dock.

\section{CONCLUSION}

Structure-based drug design tool is an effective route of drug discovery. From this, an idea to get the alternative for the existing drugs which have adverse effect after a prolonged period. Natural inhibitory compounds have no adverse effects with highest binding energy and affinity to bind the molecular markers. The molecular docking studies of the phytoligands with glycogen synthase kinase showed the excellent inhibitory potential. In conclusion, Gymnemic acid, Trigonelline, and Ferulic acid have the potential to maintain normoglycemia via the inhibition of 
GSK-3 activity. This study might be valuable for the development of active pharmacophore discovery and offering novel insights

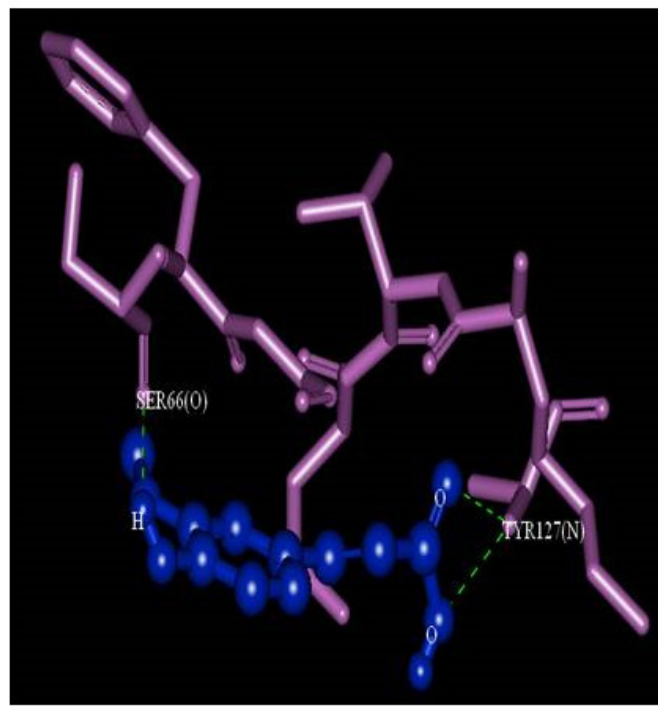

Fig. 13: Hydrogen bonds interaction between GSK3 $\beta$ and ferulic acid using Accelrys Discovery Studio Visualizer.

Table 7: The overall docking energy for Gymnemic acid, Trigonelline and Ferulic acid with Glycogen synthase kinase $3 \alpha$ and $3 \beta$.

\begin{tabular}{|c|c|c|c|}
\hline \multirow{2}{*}{ S. No } & \multirow{2}{*}{ Phytoligands } & \multicolumn{2}{|c|}{ Docking Energy (Kcal/Mol) } \\
\hline & & GSK-3a & GSK-3及 \\
\hline 1 & Gymnemic acid & -5.33 & -4.69 \\
\hline 2 & Trigonelline & -6.85 & -5.51 \\
\hline 2 & Ferulic acid & -7.0 & -5.05 \\
\hline
\end{tabular}

Table 8: Inhibition constant of Gymnemic acid, Trigonelline and Ferulic acid with Glycogen synthase kinase $3 \alpha$ and $3 \beta$.

\begin{tabular}{ccc}
\hline \multirow{2}{*}{ Phytoligands } & \multicolumn{2}{c}{ Inhibition Constant $(\boldsymbol{\mu M})$} \\
\cline { 2 - 3 } & GSK-3 $\boldsymbol{\alpha}$ & $\mathbf{G S K - 3 \beta}$ \\
\hline Gymnemic acid & 124.16 & 362.83 \\
Trigonelline & 9.5 & 92.5 \\
Ferulic acid & 7.4 & 197.29 \\
\hline
\end{tabular}

Table 9: Intermolecular energies of Gymnemic acid, Trigonelline and Ferulic acid with Glycogen synthase kinase $3 \alpha$ and $3 \beta$.

\begin{tabular}{|c|c|c|}
\hline \multirow{2}{*}{ Phytoligands } & \multicolumn{2}{|c|}{ Intermolecular energies (kcal/mol) } \\
\hline & GSK-3a & GSK-3 $\beta$ \\
\hline Gymnemic acid & -10.4 & -9.76 \\
\hline Trigonelline & -7.15 & -5.8 \\
\hline Ferulic acid & -8.49 & -6.55 \\
\hline
\end{tabular}

\section{ACKNOWLEDGMENT}

The Research Fellowship of the University Grant Commission (UGC), New Delhi, India, to the Ms. V. Roshana Devi in the form of UGC-BSR-RF is gratefully acknowledged.

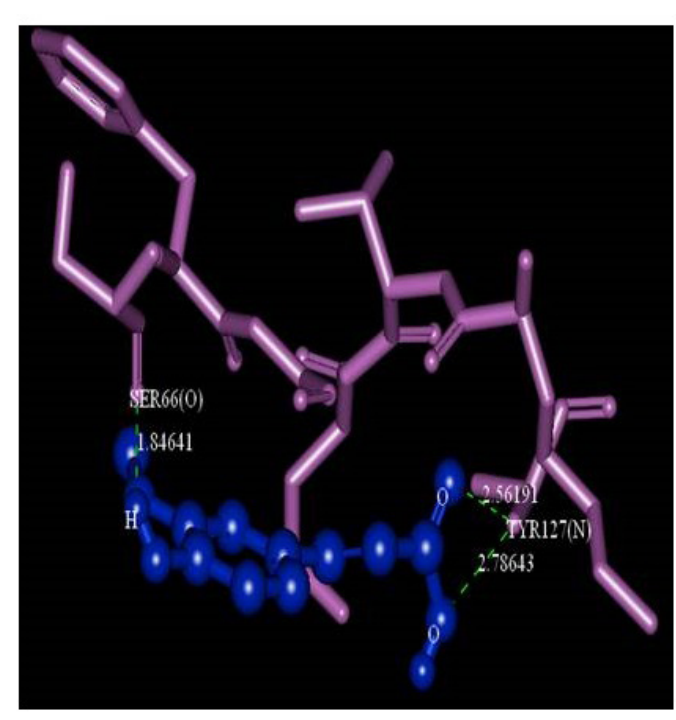

into the therapeutics for diabetes mellitus.

\section{CONFLICT OF INTEREST}

The authors declare that there is no conflict of interest.

\section{REFERENCES}

Ahmed D, Kumar V, Sharma M, Verma A. "Target guided isolation, in-vitro antidiabetic, antioxidant activity and molecular docking studies of some flavonoids from Albizzia Lebbeck Benth. bark". BMC Complementary and Alternative Medicine, 2014; 14:1-155.

Atanasov AG, Waltenberger B, Pferschy-Wenzig EM, Linder T, Wawrosch C, Uhrin P, Temml V, Wang L, Schwaiger S, Heiss EH, Rollinger JM, Schuster D, Breuss JM, Bochkov V, Mihovilovic MD, Kopp B, Bauer R, Dirsch VM, Stuppner H. Discovery and resupply of pharmacologically active plant-derived natural products: A review. Biotechnol Adv. 2015; 33(8):1582-1614.

Bissantz C, Folkers G, Rognan D. Protein-based virtual screening of chemical databases: Evaluation of different docking/scoring combinations. J Med Chem, 2000; 43:4759-4767.

Cross DA, Alessi DR, Cohen P, Andjelkovich M, Hemmings BA. Inhibition of glycogen synthase kinase-3 by insulin mediated by protein kinase B. Nature, 1995; 378:785-789.

Eldar-Finkelman H, Schreyer SA, Shinohara MM, LeBoeuf RC, Krebs EG. Increased glycogen synthase kinase-3 activity in diabetes- and obesity prone C57BL/6J mice. Diabetes, 1999; 48:1662-1666.

Embi N, Rylatt DB, Cohen P. Glycogen synthase kinase-3 from rabbit skeletal muscle. Separation from cyclic-AMP dependent protein kinase and phosphorylase kinase. Eur J Biochem, 1980; 107:519-527.

Kitchen DB, Decornez H, Furr JR, Bajorath J. Docking and scoring in virtual screening for drug discovery: methods and applications. Nature reviews Drug discovery, 2004; 3(11):935-49.

Lawlor MA, Alessi DR. PKB/Akt: a key mediator of cell proliferation, survival and insulin responses? J Cell Sci, 2001; 114:29032910.

Lengauer T, Rarey M. Computational methods for biomolecular docking. Curr Opin Struct Biol, 1996; 6(3):402-6.

Liu K, Abdullah AA, Huang M, Nishioka T, Altaf-Ul-Amin M, Kanaya S. Novel Approach to Classify Plants Based on Metabolite-Content Similarity. Biomed Res Int, 2017; 2017:5296729. 
Madeswaran A, Umamaheswari M, Asokkumar K, Sivashanmugam T, Subhadradevi V, Jagannath P. Docking studies: In silico lipoxygenase inhibitory activity of some commercially available flavonoids. Bangladesh J Pharmacol, 2011; 6:133-138.

Madeswaran et al., In silico docking studies of phosphodiesterase inhibitory activity of commercially available flavonoids. Oriental Pharmacy and Experimental Medicine, 2012; 12(4):301-306.

Middha SK, Goyal AK, Faizan SA, Sanghamitra N, Basistha $\mathrm{BC}$, Usha T. In silico-based combinatorial pharmacophore modelling and docking studies of GSK-3 $\beta$ and GK inhibitors of Hippophae. J. Biosci, $2013 ; 38: 805-814$.

Mohammad MK, Al-Masri IM, Taha MO, Al-Ghussein MA, Alkhatib HS, Najjar S, Bustanji Y. Olanzapine inhibits glycogen synthase kinase-3beta: An investigation by docking simulation and experimental validation. Eur J Pharmacol, 2008; 584:185-191.

Nikoulina SE, Ciaraldi TP, Mudaliar S, Mohideen P, Carter L, Henry RR. Potential role of glycogen synthase kinase- 3 in skeletal muscle insulin resistance of type 2 diabetes. Diabetes, 2000; 49:263-271.

Ota A, Ulrich NP. An Overview of Herbal Products and Secondary Metabolites Used for Management of Type Two Diabetes. Front Pharmacol, 2017; 8:436.

Roshana Devi V, Subramanian SP. Aldose reductase inhibitory effect of Gymnemic acid, Trigonelline and Ferulic acid - An In silico approach. International Journal of Pharmacognosy and Phytochemical Research, 2017; 9(1):31-39.

Sangu S, Vema A, Bigala R. 3D-QSAR and molecular docking studies of quinazoline derivatives as glycogen synthase kinase-3 $\beta$ (Gsk-3 $\beta$ ) inhibitors. Der Pharmacia Letter, 2014; 6 (6):289-296.
Shen W, Yan-Hua Lu. Molecular docking of citrus flavonoids with some targets related to diabetes. Bangladesh J Pharmacol, 2013; $8: 156-170$

Srinivasan S, Ohsugi M, Liu Z, Fatrai S, Bernal-Mizrachi E, Permutt MA. Endoplasmic reticulum stress-induced apoptosis is partly mediated by reduced insulin signaling through phosphatidylinositol 3-kinase/Akt and increased glycogen synthase kinase-3beta in mouse insulinoma cells. Diabetes, 2005; 54:968-975.

Stambolic V, Woodgett JR. Mitogen inactivation of glycogen synthase kinase- $3 \mathrm{~b}$ in intact cells via serine 9 phosphorylation. Biochem $\mathrm{J}$, 1994; 303:701-704.

Trott O, Olson AJ. Auto Dock Vina: improving the speed and accuracy of docking with a new scoring function, efficient optimization and multithreading. J Comput Chem, 2010; 31:455-461.

Wauwe JV, Haefner B. Glycogen synthase kinase-3 as drug target: from wallflower to center of attention. Drug News Perspect, 2003; $16: 557-565$.

How to cite this article:

Devi VR, Sharmila C, Subramanian S. Molecular Docking Studies Involving the Inhibitory Effect of Gymnemic Acid, Trigonelline and Ferulic Acid, the Phytochemicals with Antidiabetic Properties, on Glycogen Synthase Kinase 3 ( $\alpha$ and ß). J App Pharm Sci, 2018; 8(04): 150-160. 